\title{
EFECTO DE LA TEMPERATURA DE CALCINACIÓN EN LAS PROPIEDADES TEXTURALES Y ESTRUCTURALES DE Fe(III)-TiO
}

\section{EFFECT OF CALCINATION TEMPERATURE ON THE TEXTURAL AND STRUCTURAL PROPERTIES OF Fe(III)-TiO,}

Juan A. Torres-Luna',2, José G. Carriazo', Nancy Rocío Sanabria-González

\section{RESUMEN}

El dopaje del dióxido de titanio $\left(\mathrm{TiO}_{2}\right)$ con iones de metales de transición, como el $\mathrm{Fe}^{3+}$, es uno de los métodos empleados para modificar las propiedades físicas y químicas de este material. En este trabajo se analizó la influencia de la temperatura de calcinación en las propiedades texturales y estructurales del dióxido de titanio dopado con $\mathrm{Fe}^{3+}, \mathrm{Fe}(\mathrm{III})-\mathrm{TiO}_{2}$, sintetizado a partir del mineral ilmenita mediante un proceso de extracción con ácido sulfúrico. El material sintetizado fue caracterizado por fluorescencia de rayos $\mathrm{X}$, difracción de rayos X, espectroscopía infrarroja e isotermas de adsorción-desorción de nitrógeno a $77 \mathrm{~K}$. El Fe(III)-TiO, sintetizado mostró la fase anatasa como único componente cristalino, con buena estabilidad térmica a $700{ }^{\circ} \mathrm{C}$, revelando la sustitución isomórfica de hierro en la estructura del $\mathrm{TiO}_{2}$. Las distribuciones de tamaños de poros del Fe(III)$\mathrm{TiO}_{2}$ tratado a 400,500, 600 y $700{ }^{\circ} \mathrm{C}$ mostraron un comportamiento monomodal con máxima población en la región de los mesoporos. El aumento de la temperatura de calcinación del Fe(III)- $\mathrm{TiO}_{2}$ condujo a la formación de estructuras sólidas con mayores radios de poros.

Palabras clave: $\mathrm{TiO}_{2}$, ilmenita, dióxido de titanio dopado con hierro(III), anatasa.

1 Estado Sólido y Catálisis Ambiental (ESCA), Departamento de Química, Facultad de Ciencias, Universidad Nacional de Colombia - Sede Bogotá, Av. Cra 30 No. 45-03, Bogotá D.C., Colombia.

2 A quien la correspondencia debe ser enviada: jatorreslu@unal.edu.co (Juan Alberto Torres Luna) 


\begin{abstract}
Doping titanium dioxide $\left(\mathrm{TiO}_{2}\right)$ with transition metal ions, such as $\mathrm{Fe}^{3+}$, is one of methods used to modify both the physical and chemical properties of this material. In this work, the influence of calcination temperature on the textural and structural properties of titanium dioxide doped with $\mathrm{Fe}^{3+}, \mathrm{Fe}(\mathrm{III})-\mathrm{TiO}_{2}$, was analyzed. The iron-doped $\mathrm{TiO}_{2}$ was synthesized from ilmenite mineral using an extraction process with sulfuric acid. The synthesized material was characterized by $\mathrm{X}$-ray fluorescence, $\mathrm{X}$-ray diffraction, IR spectroscopy and $\mathrm{N}_{2}$ adsorption-desorption isotherms at $77 \mathrm{~K}$. Fe(III)- $\mathrm{TiO}_{2}$ showed the anatase phase as a unique crystalline component, having a good thermal stability at $700{ }^{\circ} \mathrm{C}$, and revealing the isomorphic substitution of iron in the $\mathrm{TiO}_{2}$ structure. Pore size distributions for $\mathrm{Fe}(\mathrm{III})-\mathrm{TiO}_{2}$ calcined at 400, 500, 600 y $700{ }^{\circ} \mathrm{C}$ showed a monomodal behavior with maximum values in the mesoporous region. Solids with higher values of pore radii were obtained by increasing the calcination temperature of $\mathrm{Fe}(\mathrm{III})-\mathrm{TiO}_{2}$.
\end{abstract}

Key words: $\mathrm{TiO}_{2}$, ilmenite, Iron(III)-doped titanium dioxide, anatase.

\title{
INTRODUCCIÓN
}

El dióxido de titanio $\left(\mathrm{TiO}_{2}\right)$ es un semiconductor que presenta tres polimorfos comunes: broquita, rutilo y anatasa, siendo esta última la de mayor actividad catalítica. El $\mathrm{TiO}_{2}$ es ampliamente usado para la degradación fotocatalítica de contaminantes recalcitrantes tanto en medio acuoso como en estado gaseoso, y se ha demostrado que el desempeño catalítico de este material mejora cuando es dopado con cationes de metales de transición, en especial con $\mathrm{Fe}^{3+}$, el cual además favorece su excitación con luz visible (Choi et al., 1994; Vargas et al., 2012). Las propiedades texturales del $\mathrm{TiO}_{2}$ juegan un papel importante en su actividad fotocatalítica, encontrándose que las estructuras mesoporosas con alta área superficial aumentan el número y la densidad de sitios redox de reacción (He et al., 2014). Adicionalmente la estructura mesoporosa del dióxido de titanio ofrece mayor estabilidad térmica que las nanopartículas de $\mathrm{TiO}_{2}$ convencionales (Yeh et al., 2014).
Las propiedades texturales, al igual que la cristalinidad y la fase cristalográfica deseada del $\mathrm{TiO}_{2}$ dependen del método de síntesis (He et al., 2014). Entre las rutas de síntesis empleadas para la preparación de $\mathrm{TiO}_{2}$ mesoporoso se encuentran los métodos solvotérmico (He et al., 2014), hidrotérmico, coprecipitación y sol-gel (Yeh et al., 2014). En el método sol-gel también se emplea copolímeros como plantilla para controlar la mesoporosidad de dicho material (Kim et al., 2007). En general, la síntesis del $\mathrm{TiO}_{2}$ implica el uso de precursores costosos, tóxicos y difíciles de manipular en el laboratorio $\left(\mathrm{TiCl}_{4}\right.$ y alcóxidos de titanio, entre otros). El presente trabajo se orienta a la síntesis de dióxido de titanio mesoporoso dopado con iones $\mathrm{Fe}^{3+}\left[\mathrm{Fe}(\mathrm{III})-\mathrm{TiO}_{2}\right]$, a través de una ruta de síntesis directa, usando el mineral ilmenita como fuente natural de titanio y de hierro, sin la necesidad de emplear reactivos adicionales para controlar la mesoporosidad del nuevo material. La ilmenita $\left(\mathrm{FeTiO}_{3} \circ \mathrm{FeO} \cdot \mathrm{TiO}_{2}\right)$ es un mineral 
de estructura hexagonal que está presente frecuentemente en rocas ígneas y en arenas negras de playas (Cornelis y Hurlbut, 1997). Este material suple el 91\% de la demanda global de minerales de titanio. Su explotación mundial en el año 2009 alcanzó 5,19 millones de toneladas (Zhang et al., 2011). Las reservas más grandes se ubican en Noruega, Finlandia, Rusia, Australia, India, Estados Unidos y Canadá. En Colombia se tienen depósitos de ilmenita en gran parte del territorio nacional (Mutis, 1983).

Los resultados de este trabajo permiten avanzar en la búsqueda de nuevos materiales basados en dióxido de titanio sustituido con iones $\mathrm{Fe}^{3+}$, con propiedades superiores de mesoporosidad y conservando la estructura de anatasa, mediante rutas de síntesis que involucran el aprovechamiento de los recursos minerales disponibles en nuestro país.

\section{MATERIALES Y METODOS}

\section{Materiales de partida}

Para la síntesis de $\mathrm{Fe}$ (III)-TiO, se utilizó el mineral ilmenita, proveniente de Mitú, departamento de Vaupés (Colombia). La muestra fue suministrada por el Servicio Geológico Colombiano.

En el proceso de extracción de las especies (polihidroxicationes) de hierro y $\mathrm{TiO}_{2}$ de la ilmenita se empleó ácido sulfúrico (Merck, 98\%), Fe en polvo (J. T. Baker), hidróxido de sodio (Mallinckrodt, 99,0\%) y agua desionizada. El $\mathrm{TiO}_{2}$ empleado como referencia correspondió a Evonik P25 (Evonik Degussa $\mathrm{GMBH}$, Alemania), el cual contiene $80 \%$ de anatasa y $20 \%$ de rutilo.

\section{Síntesis del Fe-TiO,}

La extracción de las especies de titanio-hierro del mineral ilmenita fue adaptada de la metodología denominada "proceso del sulfato" (Chernet, 1999). Para ello, $18 \mathrm{~g}$ del mineral ilmenita se mezclaron con $32 \mathrm{~mL}$ de una solución acuosa de ácido sulfúrico al
$80 \%$ y la suspensión se agitó durante 1,5 horas a una temperatura de $110^{\circ} \mathrm{C}$. La extracción se lleva a cabo en un balón de tres bocas provisto con un sistema de reflujo y control de temperatura. Para evitar escapes de vapores ácidos al ambiente, al sistema de reflujo (condensador) se acopla una trampa de gases formada por una disolución de hidróxido de sodio.

La ilmenita $\left(\mathrm{FeTiO}_{3}\right)$ se digirió con la solución acuosa de ácido sulfúrico para producir una solución acuosa de sulfato de titanilo $\left[(\mathrm{TiO}) \mathrm{SO}_{4}\right]$ y sulfato ferroso $\left(\mathrm{FeSO}_{4}\right)$ de acuerdo con la siguiente ecuación (Chernet, 1999; Sasikumar et al., 2004):

$$
\mathrm{FeTiO}_{3}+2 \mathrm{H}_{2} \mathrm{SO}_{4} \rightarrow \mathrm{TiOSO}_{4}+\mathrm{FeSO}_{4}+2 \mathrm{H}_{2} \mathrm{O}
$$

El ion férrico generado durante el proceso es reducido a ion ferroso mediante la adición de un exceso Fe (metálico) en polvo. Luego, la mezcla se enfrió a 10 ${ }^{\circ} \mathrm{C}$ durante 24 horas y los cristales de sulfato ferroso hidratado y el exceso de hierro en polvo adicionado fueron separados del medio de reacción. A la solución resultante se le ajustó el pH a 1,2 y luego se le sometió a un proceso de hidrólisis durante una hora, con el fin de precipitar las especies polihidroxicatiónicas de titanio-hierro $\left(\left[\mathrm{Fe}_{x} \mathrm{Ti}_{(8-x)} \mathrm{O}_{8}(\mathrm{OH})_{12}\left(\mathrm{H}_{2} \mathrm{O}\right)_{m}\right]^{\mathrm{n+}}\right.$, donde $n=4-x)$. El sólido resultante fue lavado con agua desionizada, secado a $60^{\circ} \mathrm{C}$ y calcinado por 2 horas, en atmosfera estática de aire, a diferentes temperaturas $\left(400,500,600\right.$ y $\left.700{ }^{\circ} \mathrm{C}\right)$, empleando una velocidad de calentamiento de $10^{\circ} \mathrm{C} / \mathrm{min}$.

\section{Técnicas de caracterización}

Los sólidos sintetizados correspondientes a $\mathrm{Fe}(\mathrm{III})-\mathrm{TiO}_{2}$ calcinado a diferentes temperaturas fueron triturados y tamizados en malla ASTM número 100, y posteriormente caracterizados por fluorescencia de rayos $X(F R X)$, difracción de rayos $X(D R X)$, espectroscopía infrarroja y adsorción-desorción de $\mathrm{N}_{2}$ a $77 \mathrm{~K}$. El análisis de FRX fue realizado en un espectrómetro Magix Pro PW-2440 Philips, equipado 
con un tubo de rodio y una potencia máxima de 4 kW. Los perfiles de DRX fueron tomados en un equipo Panalytical X'Pert PRO MPD, con ánodo de $\mathrm{Cu}$ (radiación $\mathrm{Ka} \lambda=1,5406 \AA$ A). Los difractogramas se tomaron mediante la técnica de polvos, a temperatura ambiente, tamaño de paso de $0,02^{\circ} 2 \theta$ y tiempo de paso $10 \mathrm{~s}$. Los espectros de infrarrojo fueron tomados en un equipo NICOLET Thermo ScientificiS10, haciendo pastillas con $\mathrm{KBr}$ (2 mg de muestra en $200 \mathrm{mg}$ de $\mathrm{KBr}$ ). Las propiedades texturales de los sólidos se determinaron mediante isotermas de adsorción-desorción de nitrógeno a 77 K, usando un equipo Micromeritics ASAP 2020 en el intervalo de presiones relativas $\left(P / P_{0}\right)$ de $1 \times 10^{-5}$ a 0,99 . Las muestras se desgasificaron previamente a $350{ }^{\circ} \mathrm{C}$ durante 4 horas. Para determinar las áreas superficiales se empleó el modelo BET (Brunauer, Emmett y Teller) en el intervalo de presiones relativas $0,05 \leq \mathrm{P} / \mathrm{P}_{0} \leq$ 0,35 . El volumen total de poro se calculó empleando el método de Gurvitsch y la distribución de tamaño de poro se determinó por el método BJH (Barrett, Joyner y Halenda) (Leofanti et al., 1998).

\section{RESULTADOS Y DISCUSIÓN}

En la Tabla 1 se muestra la composición química del mineral ilmenita y el sólido $\mathrm{Fe}(\mathrm{III})-\mathrm{TiO}_{2}$ calcinado a $400{ }^{\circ} \mathrm{C}$. La ilmenita presentó un alto contenido de titanio y de hierro, y un bajo porcentaje impurezas de Si y Al. El Fe(III)-TiO, sintetizado a partir de la ilmenita mostró un alto contenido de titanio frente a la cantidad de hierro incorporado (0,75\% molar), así como impurezas de azufre asociadas al proceso de extracción con ácido sulfúrico.

En la Figura 1 se aprecian los difractogramas del mineral ilmenita, el $\mathrm{Fe}(\mathrm{III})-\mathrm{TiO}_{2}$ calcinado a diferentes temperaturas y el $\mathrm{TiO}_{2}$ empleado como referencia (Evonik P25). El perfil de DRX de la ilmenita presenta las señales características para el titanato de hierro $\left({ }^{\circ} 2 \theta=32,47 ; 35,24 ; 48,70 ; 23,79\right.$ y 53,93) (Anthony et al., 2001; Ganesh et al., 2012). En los difractogramas del $\mathrm{Fe}(\mathrm{III})-\mathrm{TiO}_{2}$ calcinado a diferentes temperaturas se observa claramente que la estructura obtenida corresponde a la fase anatasa $\left({ }^{\circ} 2 \theta=25,3 ; 37,8 ; 48,1\right.$; $54,4)$ y aunque el análisis químico confirmó la presencia de hierro, no se observan señales propias de óxidos de hierro. Lo anterior sugiere una sustitución isomórfica de $\mathrm{Fe}^{3+}$ en la estructura del $\mathrm{TiO}_{2}$. Al aumentar la temperatura de calcinación en el sólido $\mathrm{Fe}(\mathrm{III})-\mathrm{TiO}_{2}$ de 400 a $700{ }^{\circ} \mathrm{C}$, las señales en el difractograma se hacen más simétricas y definidas (agudas), lo que está relacionado con una mayor cristalinidad del material. Al incrementar la temperatura de calcinación, aumenta la difusión de los átomos dentro del material, lo que permite una mayor organización estructural y por tanto una red cristalina mejor definida (Yeh et al., 2014).

El ensanchamiento y la disminución de la intensidad de las señales en los difractogramas de $\mathrm{Fe}(\mathrm{III})-\mathrm{TiO}_{2}$ a temperaturas inferiones a los $600{ }^{\circ} \mathrm{C}$ es

Tabla 1. Composición química de la ilmenita y el sólido $\mathrm{Fe}(\mathrm{III})-\mathrm{TiO}_{2}$

\begin{tabular}{ccccccc}
\hline Sólidos & $\mathrm{Ti}(\%)$ & $\mathrm{Fe}(\%)$ & Al (\%) & Si (\%) & S (\%) & $\begin{array}{c}\text { Fe } \\
\text { (\% molar) }\end{array}$ \\
\hline Ilmenita & 27,52 & 34,61 & 0,32 & 0,70 & - & - \\
\hline $\begin{array}{r}\mathrm{Fe}(\mathrm{III})-\mathrm{TiO}_{2} \\
\left(\mathbf{4 0 0} \mathbf{C}^{\circ}\right.\end{array}$ & 57,00 & 0,50 & - & - & 2,33 & 0,75 \\
\hline
\end{tabular}


atribuido a la posible incorporación del hierro (sustitución isomórfica) en la red cristalina del dióxido de titanio (Tong et al., 2008; Jamalluddin y Abdullah, 2011; Rauf et al., 2011; Babić et al., 2012; Varga et al., 2012), lo cual genera variaciones en el valor de la energía de red de la estructura sustituida (anatasa), dificultando el proceso de cristalización (Carriazo et al., 2010). Los resultados encontrados en el presente trabajo apoyan la veracidad de dicha hipótesis, puesto que el tratamiento térmico modificó la forma de las señales, pero no condujo a la formación de fases cristalográficas diferentes a la anatasa; aunque en otros trabajos se ha señalado la presencia de la fase rutilo a partir de $500{ }^{\circ} \mathrm{C}$ y $600{ }^{\circ} \mathrm{C}$ (Yeh et al., 2014). La sustitución isomórfica de $\mathrm{Fe}^{3+}$ por $\mathrm{Ti}^{4+}$ en la red de anatasa es posible de acuerdo a las reglas de Hume-Rothery (Shackelford, 1998), sin embargo la elevada similitud de los radios iónicos $(0,74 \AA$ para $\mathrm{Ti}^{4+}$ y $0,78 \AA$ para $\mathrm{Fe}^{3+}$ ) hace difícil la detección de cualquier desplazamiento de las posiciones de las señales en DRX (Carriazo et al., 2010).

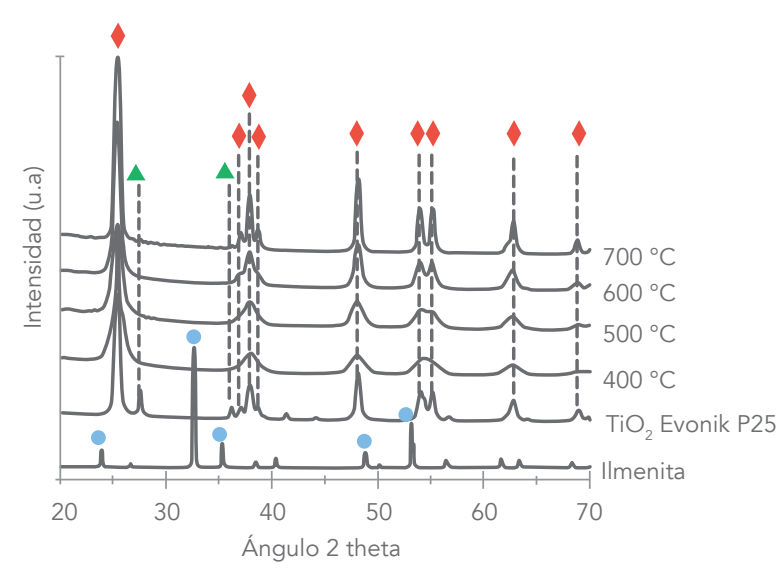

Figura 1. Patrones de difracción de rayos $X$ de la ilmenita, $\mathrm{Fe}(\mathrm{IIII})-\mathrm{TiO}_{2}$ calcinado a diferentes temperaturas $\left(400,500,600\right.$ y $\left.700^{\circ} \mathrm{C}\right)$ y del $\mathrm{TiO}_{2}$ Evonik P25 ( ilmenita, anatasa, $\Delta$ rutilo).

Los espectros infrarrojo (IR) del mineral ilmenita, el Fe(III)-TiO, extraido del mineral ilmenita calcinado a diferentes temperaturas, y el $\mathrm{TiO}_{2}$ empleado como referencia (Evonik P25) se muestran en la Figura 2. La ilmenita presenta una banda ancha en el intervalo comprendido entre 450 y $650 \mathrm{~cm}^{-1}$, correspondiente a vibraciones características de enlaces $\mathrm{Ti}-\mathrm{O}-\mathrm{Ti}$ (Navío et al., 1999). El Fe(III)-TiO 2 calcinado a diferentes temperaturas presenta señales características del dióxido de titanio de referencia (Evonik P25), así como una banda a $1130 \mathrm{~cm}^{-1}$ asociada a vibraciones características de grupos sulfatos (Stuart, 2004), evidenciados por fluorescencia de rayos $X$. Esta señal que aparece en el $\mathrm{Fe}(\mathrm{III})-\mathrm{TiO}_{2}$ calcinado a $400{ }^{\circ} \mathrm{C}$ desaparece con el tratamiento térmico a $700{ }^{\circ} \mathrm{C}$. Asimismo, se observa un hombro alrededor de 1200 $\mathrm{cm}^{-1}$ atribuido a las vibraciones Ti-O-Ti (Ganesh et al., 2012). Alrededor de $3400 \mathrm{~cm}^{-1}$ se observa una banda relacionada a las vibraciones de estiramiento de enlaces $\mathrm{O}-\mathrm{H}$ de agua adsorbida, y en 1626 $\mathrm{cm}^{-1}$ se obtienen las flexiones de dichos enlaces (Ganesh et al., 2012). La diferencia encontrada entre los espectros del mineral ilmenita y los del Fe(III)- $\mathrm{TiO}_{2}$, y la similitud de estos últimos con el espectro del $\mathrm{TiO}_{2}$ Evonik P25, confirma el éxito del proceso de extracción del $\mathrm{Fe}(\mathrm{III})-\mathrm{TiO}_{2}$ a partir del mineral ilmenita.

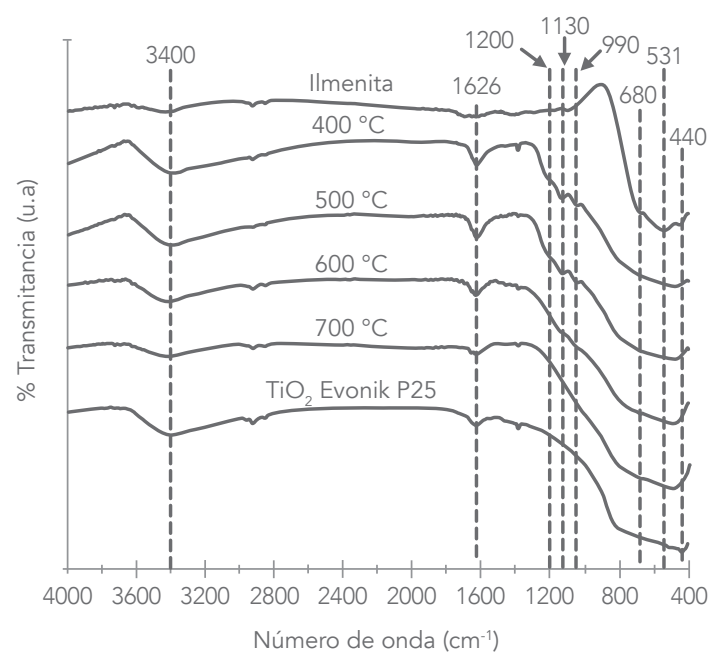

Figura 2. Espectros infrarrojos de la ilmenita, $\mathrm{Fe}(\mathrm{III})-\mathrm{TiO}_{2}$ calcinado a diferentes temperaturas y $\mathrm{TiO}_{2}$ Evonik P25 
La Figura 3 muestra las isotermas de adsorcióndesorción de $\mathrm{N}_{2}$ para el sólido $\mathrm{Fe}$ (III)- $\mathrm{TiO}_{2}$ sometido a diferentes temperaturas de calcinación, así como la del $\mathrm{TiO}_{2}$ de referencia. Las isotermas de los sólidos correspondientes a Fe(III)- $\mathrm{TiO}_{2}$ son de tipo IV (Leofanti et al., 1998; Jamalluddin y Abdullah, 2011) con histéresis tipo H2. La histéresis H2 está asociada, en este caso, a sólidos formados por agregados de partículas de tamaño y forma no uniformes (Leofanti et al., 1998). Al aumentar la temperatura de calcinación del $\mathrm{Fe}(\mathrm{III})-\mathrm{TiO}_{2}$ se aprecia una disminución en los volúmenes adsorbidos. Adicionalmente, la histéresis

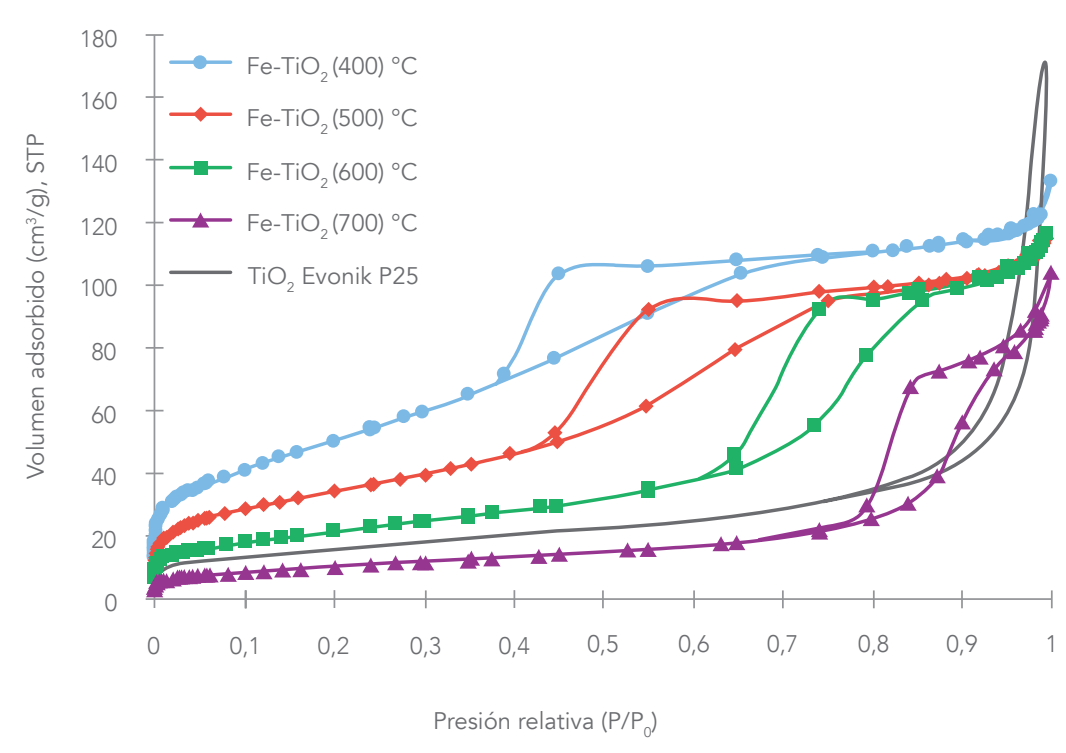

Figura 3. Isotermas de adsorción-desorción de $\mathrm{N}_{2}$ para Fe(III)-TiO 2 calcinado a diferentes temperaturas y el TiO $\mathrm{TV}_{2}$ enik P25

tiende a ser de tipo $\mathrm{H} 1$, similar a la que presenta el $\mathrm{TiO}_{2}$ Evonik P25, la cual es atribuida a poros con tamaño y forma uniforme (Leofanti et al., 1998).

Las distribuciones de tamaños de poros obtenidas para el $\mathrm{Fe}(\mathrm{III})-\mathrm{TiO}_{2}$ sometido a diferentes temperaturas de calcinación y para el $\mathrm{TiO}_{2}$ de referencia se muestran en la Figura 4. Para el Fe(III)$\mathrm{TiO}_{2}$ calcinado a $400,500,600$ y $700^{\circ} \mathrm{C}$ se observa una distribución monomodal cuyo radio de poro se encuentra dentro la clasificación de mesoporos de la IUPAC. Además, el incremento en la temperatura de calcinación condujo al aumento del radio de poro promedio. En general, el $\mathrm{TiO}_{2}$ de referencia presenta una distribución de tamaños de poros más heterogénea que el $\mathrm{Fe}(\mathrm{III})-\mathrm{TiO}_{2}$ extraído de la ilmenita. Por tanto, el método de extracción de $\mathrm{Fe}(\mathrm{III})-\mathrm{TiO}_{2}$ a partir de la ilmenita condujo a la obtención de una estructura mesoporosa, a la cual se le puede controlar el tamaño de poro variando la temperatura de calcinación, ya que al someter este tipo de material a un tratamiento térmico cada vez mayor los grupos hidroxilo presentes son retirados de la estructura. Ello altera la estabilidad del material e induce al reordenamiento del mismo, llevándose a cabo la formación de cavidades de mayor tamaño como producto de la destrucción de poros pequeños, lo cual se refleja en la distribución de tamaño de poros. 


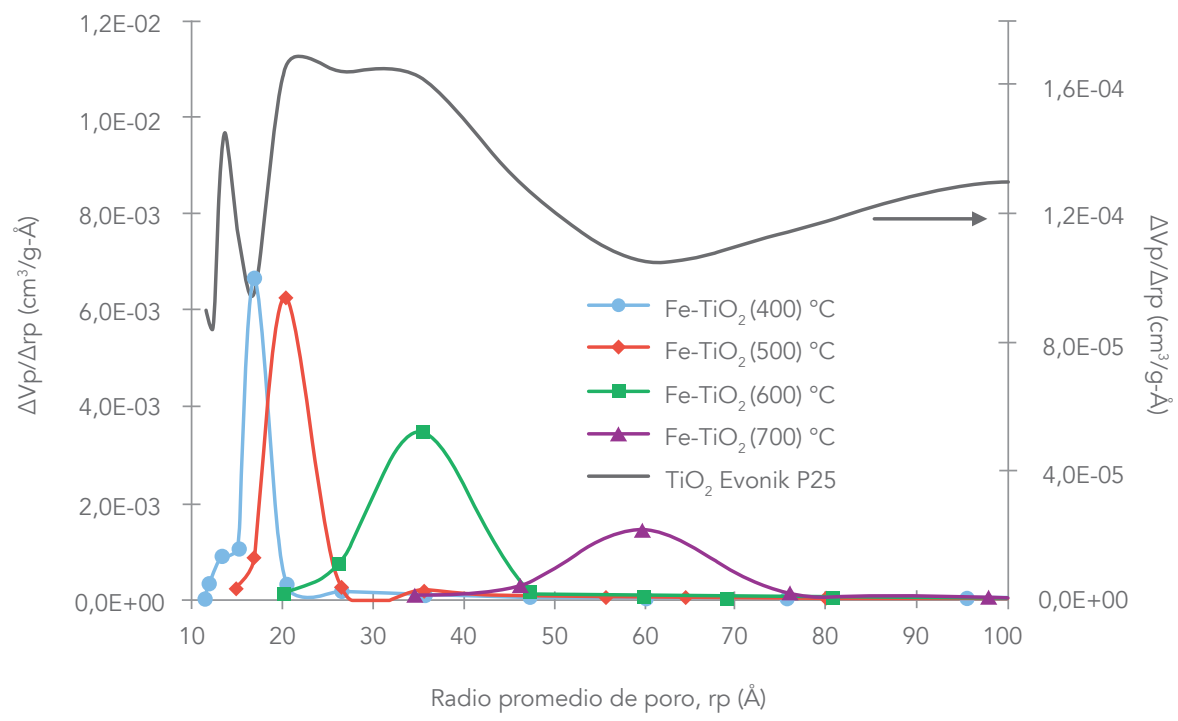

Figura 4. Distribución de tamaños de poros de $\mathrm{Fe}(\mathrm{IIII})-\mathrm{TiO}_{2}$ calcinado a diferentes temperaturas y $\mathrm{TiO}_{2}$ Evonik P25

En la Tabla 2 se muestran los valores de área superficial, volumen total de poro y radio promedio de poro para los diferentes sólidos. Las áreas superficiales $\mathrm{BET}$ para $\mathrm{Fe}(\mathrm{III})-\mathrm{TiO}_{2}$ calcinado a 400,500 y $600^{\circ} \mathrm{C}$ son superiores a la del $\mathrm{TiO}_{2}$ Evonik P25, la cual es de $55 \mathrm{~m}^{2} / \mathrm{g}$. Este resultado puede estar asociado con la incorporación de hierro, ya que se ha publicado que el dopaje de $\mathrm{TiO}_{2}$ con $\mathrm{Fe}^{3+}$ aumenta el área superficial (Wang et al., 2006). En general, el área superficial de $\mathrm{Fe}(\mathrm{III})-\mathrm{TiO}_{2}$ disminuye al aumentar la temperatura de calcinación, lo cual está relacionado con la generación de poros más grandes, producto del tratamiento térmico y el posible colapso de la estructura microporosa.

Tabla 2. Resumen de las propiedades texturales de $\mathrm{Fe}(\mathrm{III})-\mathrm{TiO}_{2}$ calcinado a diferentes temperaturas y del $\mathrm{TiO}_{2} \mathrm{Evonik}_{\mathrm{P} 25}$

\begin{tabular}{cccc}
\hline Sólidos & $\begin{array}{c}\text { Área superficial } \\
\mathbf{S}_{\mathrm{BET}}\left(\mathbf{m}^{2} \mathbf{g}^{-1}\right)\end{array}$ & $\begin{array}{c}\mathbf{V}_{\text {total }} \\
\left(\mathbf{c m}^{3} \mathbf{g}^{-1}\right)\end{array}$ & $\begin{array}{c}\text { Radio promedio } \\
\text { de poro (Å) }\end{array}$ \\
\hline $\mathrm{TiO}_{2}$ & 55 & 0,2031 & - \\
\hline $\mathrm{Fe}(\mathrm{IIII})-\mathrm{TiO}_{2}\left(\mathbf{4 0 0}{ }^{\circ} \mathrm{C}\right)$ & 187 & 0,2048 & 17 \\
\hline $\mathrm{Fe}(\mathrm{III})-\mathrm{TiO}_{2}\left(500^{\circ} \mathrm{C}\right)$ & 122 & 0,1744 & 20 \\
\hline $\mathrm{Fe}(\mathrm{III})-\mathrm{TiO}_{2}\left(600^{\circ} \mathrm{C}\right)$ & 75 & 0,1735 & 35 \\
\hline $\mathrm{Fe}(\mathrm{III})-\mathrm{TiO}_{2}\left(\mathbf{7 0 0}{ }^{\circ} \mathrm{C}\right)$ & 35 & 0,1383 & 60 \\
\hline
\end{tabular}




\section{CONCLUSIONES}

Se logró sintetizar dióxido de titanio dopado con $\mathrm{Fe}^{3+}, \mathrm{Fe}(\mathrm{III})-\mathrm{TiO}_{2}$, con bajo contenido de $\mathrm{Fe}(0,75 \%$ molar), empleando una ruta directa de transformación del mineral ilmenita. El Fe(III)-TiO ${ }_{2}$ calcinado a diferentes temperaturas $\left(400,500600\right.$ y $\left.700{ }^{\circ} \mathrm{C}\right)$ presentó una estructura mesoporosa, con la posibilidad de regular el tamaño de poros y el área superficial variando la temperatura de calcinación. Los perfiles de difracción de $\mathrm{Fe}(\mathrm{III})-\mathrm{TiO}_{2}$ calcinado a temperaturas diferentes mostraron la presencia de anatasa como única fase cristalográfica, inclusive cuando el material es calcinado a $700{ }^{\circ} \mathrm{C}$. El dopaje del $\mathrm{TiO}_{2}$ con $\mathrm{Fe}^{3+}$ aumenta la estabilidad de la fase anatasa y retarda la transición de dicha fase a rutilo.

\section{AGRADECIMIENTOS}

Los autores agradecen a la Universidad Nacional de Colombia-Bogotá por el apoyo logístico en el desarrollo del presente trabajo.

Para el Fe(III)- $\mathrm{TiO}_{2}$ calcinado a 400, 500, 600 y $700^{\circ} \mathrm{C}$ se observa una distribución monomodal cuyo radio de poro se encuentra dentro la clasificación de mesoporos de la IUPAC. Además, el incremento en la temperatura de calcinación condujo al aumento del radio de poro promedio. 


\section{REFERENCIAS}

1. Anthony JW, Bideaux RA, Bladh KW y Nichols MC. 2001. Handbook of Mineralogy. Mineralogical Society of America, Chantilly, VA, USA, 3960p

2. Babić B, Gulicovski J, Dohčević-Mitrović Z, Bučevac D, Prekajski M, Zagorac J y Matović B. 2012. Synthesis and characterization of $\mathrm{Fe}^{3+}$ doped titanium dioxide nanopowders. Ceramics International 38:635-640.

3. Carriazo JG, Moreno-Forero M, Molina RA y Moreno S. 2010. Incorporation of titanium and titanium-iron species inside a smectite-type mineral for photocatalysis. Applied Clay Science, 50:401-408.

4. Chernet T. 1999. Applied mineralogical studies on Australian sand ilmenite concentrate with special reference to its behaviour in the sulphate process. Minerals Engineering, 12:485-495.

5. Choi W, Termin A y Hoffmann MR. 1994. The role of metal ion dopants in quantumsized $\mathrm{TiO}_{2}$ : correlation between photoreactivity and charge carrier recombination dynamics. The Journal of Physical Chemistry, 98:13669-13679.
6. Cornelis K y Hurlbut C. 1997. Manual de Mineralogía. Ed. 4. Reverté, Barcelona, 392p.

7. Ganesh I, Kumar PP, Gupta AK, Sekhar PSC, Radha K, Padmanabham G y Sundararajan G. 2012. Preparation and characterization of $\mathrm{Fe}$ doped $\mathrm{TiO}_{2}$ powers for solar light response and photocatalytic applications. Processing and Application of Ceramin, 6:21-36.

8. He F, Li J, Li T y Li G. 2014. Solvothermal synthesis of mesoporous $\mathrm{TiO}_{2}$ : The effect of morphology, size and calcination progress on photocatalytic activity in the degradation of gaseous benzene. Chemical Engineering Journal, 237:312-321.

9. Jamalluddin NA y Abdullah AZ. 2011. Reactive dye degradation by combined Fe(III)/ $\mathrm{TiO}_{2}$ catalyst and ultrasonic irradiation: Effect of $\mathrm{Fe}(\mathrm{III})$ loading and calcination temperature. Ultrasonics Sonochemistry, 18:669-678.

10. Kim DS, Han SJ y Kwak S-Y. 2007. Synthesis and photocatalytic activity of mesoporous $\mathrm{TiO}_{2}$ with the surface area, crystallite size, and pore size. Journal of Colloid and Interface Science, 316:85-91. 
11. Leofanti G., Padovan M, Tozzola G y Venturelli B. 1998. Surface area and pore texture of catalysts. Catalysis Today, 41:207-219.

12. Mutis V. 1983. Cátalogo de los yacimientos, prospecto y manifestaciones minerales de Colombia. Ingeominas, Bogotá, 462p.

13. Navío JA, Colón G, Macías M, Real C y Litte MI. 1999. Iron-doped titania semiconductor powders prepared by a sol-gel method. Part I: synthesis and characterization. Applied Catalysis A: General, 177:111-120.

14. Rauf MA, Meetani MA y Hisaindee S. 2011. An overview on the photocatalytic degradation of azo dyes in the presence of $\mathrm{TiO}_{2}$ doped with selective transition metals. Desalination 276:13-27.

15. Sasikumar C, Rao DS, Srikanth S, Ravikumar B, Mukhopadhyay NK y Mehrotra SP. 2004. Effect of mechanical activation on the kinetics of sulfuric acid leaching of beach sand ilmenite from Orissa, India. Hydrometallurgy 75:189-204.

16. Shackelford J. 1998. Introducción a la Ciencia de Materiales para Ingenieros, Ed. 4. Prentice Hall, Madrid, 675p.

17. Stuart B. 2004. Infrarred Spectroscopy: Fundamental and Aplications. Wiley, 203p.
18. Tong T, Zhang J, Tian B, Chen F y He D. 2008. Preparation of $\mathrm{Fe}^{3+}$-doped $\mathrm{TiO}_{2}$ catalysts by controlled hydrolysis of titanium alkoxide and study on their photocatalytic activity for methyl orange degradation. Journal of Hazardous Materials 155: 572-579.

19. Vargas X, Tauchert E, Marin J-M, Restrepo G, Dillert R y Bahnemann D. 2012. Fe-doped titanium dioxide synthesized: Photocatalytic activity and mineralization study for azo dye. Journal of Photochemistry and Photobiology A: Chemistry, 243:17- 22.

20. Wang Y, Jiang Z-H y Yang F-J. 2006. Effect of Fe-doping on the pore structure of mesoporous titania. Materials Science and Engineering B, 134:76-79.

21. Yeh S-W, Ko H-H, Chiang S-M, Chen Y-L, Lee J-H, Wen C-M y Wang M-C. 2014. Characteristics and properties of a novel in situ method of synthesizing mesoporous $\mathrm{TiO}_{2}$ nanopowders by a simple coprecipitation process without adding surfactant. Journal of Alloys and Compounds, 613:107-116.

22. Zhang W, Zhu Z y Cheng CY. 2011. A literature review of titanium metallurgical processes. Hydrometallurgy, 108:177-188. 